\title{
Solar stereoscopy - where are we and what developments do we require to progress?
}

\author{
T. Wiegelmann ${ }^{1}$, B. Inhester ${ }^{1}$, and L. Feng ${ }^{1,2}$ \\ ${ }^{1}$ Max-Planck-Institut für Sonnensystemforschung, Max-Planck-Strasse 2, 37191 Katlenburg-Lindau, Germany \\ ${ }^{2}$ Purple Mountain Observatory, Chinese Academy of Sciences, 210008, Nanjing, China
}

Received: 19 May 2009 - Revised: 13 July 2009 - Accepted: 17 July 2009 - Published: 23 July 2009

\begin{abstract}
Observations from the two STEREO-spacecraft give us for the first time the possibility to use stereoscopic methods to reconstruct the 3-D solar corona. Classical stereoscopy works best for solid objects with clear edges. Consequently an application of classical stereoscopic methods to the faint structures visible in the optically thin coronal plasma is by no means straight forward and several problems have to be treated adequately: 1) First there is the problem of identifying one-dimensional structures - e.g. active region coronal loops or polar plumes- from the two individual EUVimages observed with STEREO/EUVI. 2) As a next step one has the association problem to find corresponding structures in both images. This becomes more difficult as the angle between STEREO-A and B increases. 3) Within the reconstruction problem stereoscopic methods are used to compute the 3-D-geometry of the identified structures. Without any prior assumptions, e.g., regarding the footpoints of coronal loops, the reconstruction problem has not one unique solution. 4) One has to estimate the reconstruction error or accuracy of the reconstructed 3-D-structure, which depends on the accuracy of the identified structures in 2-D, the separation angle between the spacecraft, but also on the location, e.g., for east-west directed coronal loops the reconstruction error is highest close to the loop top. 5) Eventually we are not only interested in the 3-D-geometry of loops or plumes, but also in physical parameters like density, temperature, plasma flow, magnetic field strength etc. Helpful for treating some of these problems are coronal magnetic field models extrapolated from photospheric measurements, because observed EUV-loops outline the magnetic field. This feature has been used for a new method dubbed "magnetic stereoscopy". As examples we show recent application to active region loops.
\end{abstract}

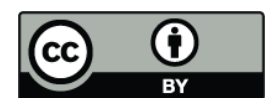

Correspondence to: T. Wiegelmann (wiegelmann@mps.mpg.de)
Keywords. Solar physics, astrophysics,and astronomy (Corona and transition region; Magnetic fields; Ultraviolet emissions)

\section{Introduction}

The Solar TErrestrial RElations Observatory (STEREO) observes for the first time simultaneously the Sun from two vantage points (see Kaiser et al., 2008) and allows a threedimensional view of the solar corona. Within this work we aim to review stereoscopic methods to reconstruct the 3-D corona and will concentrate mainly on structures like active region loops and polar plumes observed with the STEREO/SECCHI instrument package (Sun Earth Connection Coronal and Heliospheric Investigation, Howard et al., 2008). Before the launch of STEREO on 26 October 2006 stereoscopic methods have been applied for example to Skylab images by Berton and Sakurai (1985) and Batchelor (1994), and to SOHO data by (Aschwanden et al., 1999, 2000). In these pre-STEREO cases the authors used the solar rotation and took images a few hours to a day apart to reconstruct the 3-D structures under the assumption that all basic features remain stationary within this time. The stationarity assumption was somewhat relaxed by introducing the concept of "dynamic stereoscopy" (Aschwanden et al., 1999), which uses the a priori information of a coplanar loop shape and a corresponding fitting procedure. In this approach the coronal magnetic field - but not necessarily the plasma - is considered to be quasi-stationary. The method takes advantage of the near-parallelity of adjacent magnetic field lines, even if the loop plasma is heated and cools down on much faster time scales than the time interval of stereoscopic correlation. Such limitations are not necessary anymore after the launch of STEREO about two and a half year ago and

Published by Copernicus Publications on behalf of the European Geosciences Union. 
within this paper we would like to give a review on what has been done in solar stereoscopy so far, which developments are currently under consideration, and planned for the future.

The key question is how we can derive the 3-D geometry and physical structure of the solar corona from images observed with the two STEREO-spacecraft? In Sect. 2 we describe a step by step guide, which contains the identification of curvi-linear structures from coronal EUV-images in Sect. 2.1, the association of the identified structures in both images from different vantage viewpoints in Sect. 2.2, the geometric 3-D stereoscopy (Sect. 2.3) and estimation of the 3-D-reconstruction error in Sect. 2.4. After these steps one has obtained the 3-D geometry of, e.g., active region loops or polar plumes and in Sect. 2.5 we outline how physical quantities like temperature and density can be found. An interesting question, which we address in Sect. 3, is how well do stereoscopic reconstructed plasma loops agree with coronal magnetic field models? Due to the high conductivity of the coronal plasma the magnetic field is outlined by the radiating plasma and in principle one has two independent data sources about the 3-D geometry of coronal loops, namely stereoscopy and magnetic field extrapolations from photospheric measurements. In Sect. 4 we address how in future stereoscopic, tomographic and self-consistent modelling approaches could be combined and diminish weaknesses of the individual approaches.

\section{Step by step guide to stereoscopy}

\subsection{Extraction of curvi-linear objects from EUV-images}

A first step for stereoscopy is to extract curve-like structures (projections of coronal loops) from observed EUV-images such as from the TRACE-image shown in Fig. 1a). A principal problem of identifying loops is that the solar corona is optically thin and the loops are faint. Visible loops are often a superposition of multiple individual loops (Schrijver et al., 1999, 2004). Figure $1 \mathrm{~b}$ shows the TRACE-image after a $7 \times 7$-boxcar smoothed image was removed from the original, which enhances the contrast. Aschwanden et al. (2008a) manually traced 210 loops as shown in panel (c). While hand-tracing might be suitable to investigate a few individual cases, this is not appropriate to study large data sets and time sequences. Several automatic feature recognition methods have been developed. The example shown in Fig. 1 has been used to compare and evaluate five automated loop segmentation methods. The output of the five codes has been compared with the hand-traced loops from Fig. 1c. This comparison revealed large differences. The codes identified between 76 and 347 loops. Among the longer and more significant loops, the various codes identified between $19 \%$ and 59\% of the corresponding 154 hand traced loops above this limit. Some codes wrongly identified noise as spurious short loops. Obviously the state-of-the-art of automatic
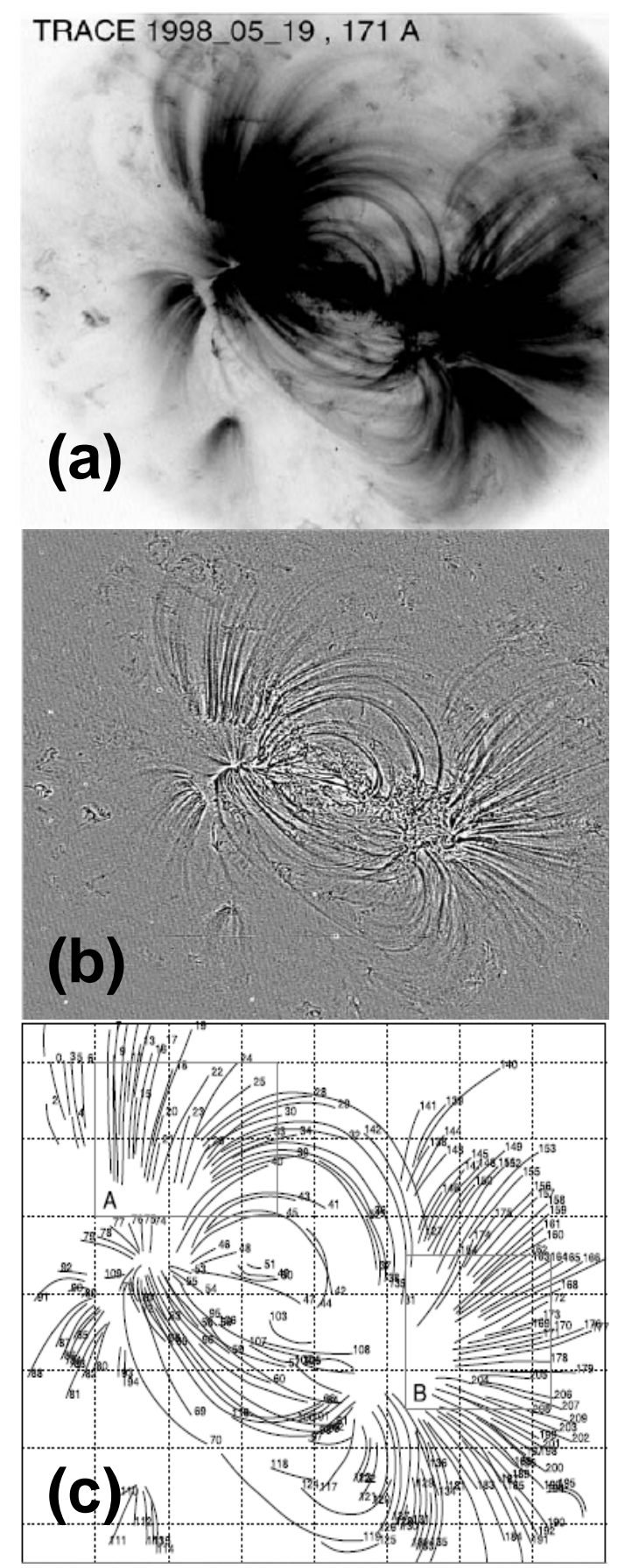

Fig. 1. Panel (a) shows an original $171 \AA$ TRACE image of 19 May 1998, panel (b) a corresponding high-pass-filtered image, where a $7 \times 7$-boxcar smoothed image was subtracted from the original. The image has been used to compare the output of five feature recognition codes with the result of 210 manually traced loop shown in panel (c) (original figures are from Aschwanden et al., 2008a, Figs. 1 and 2). 

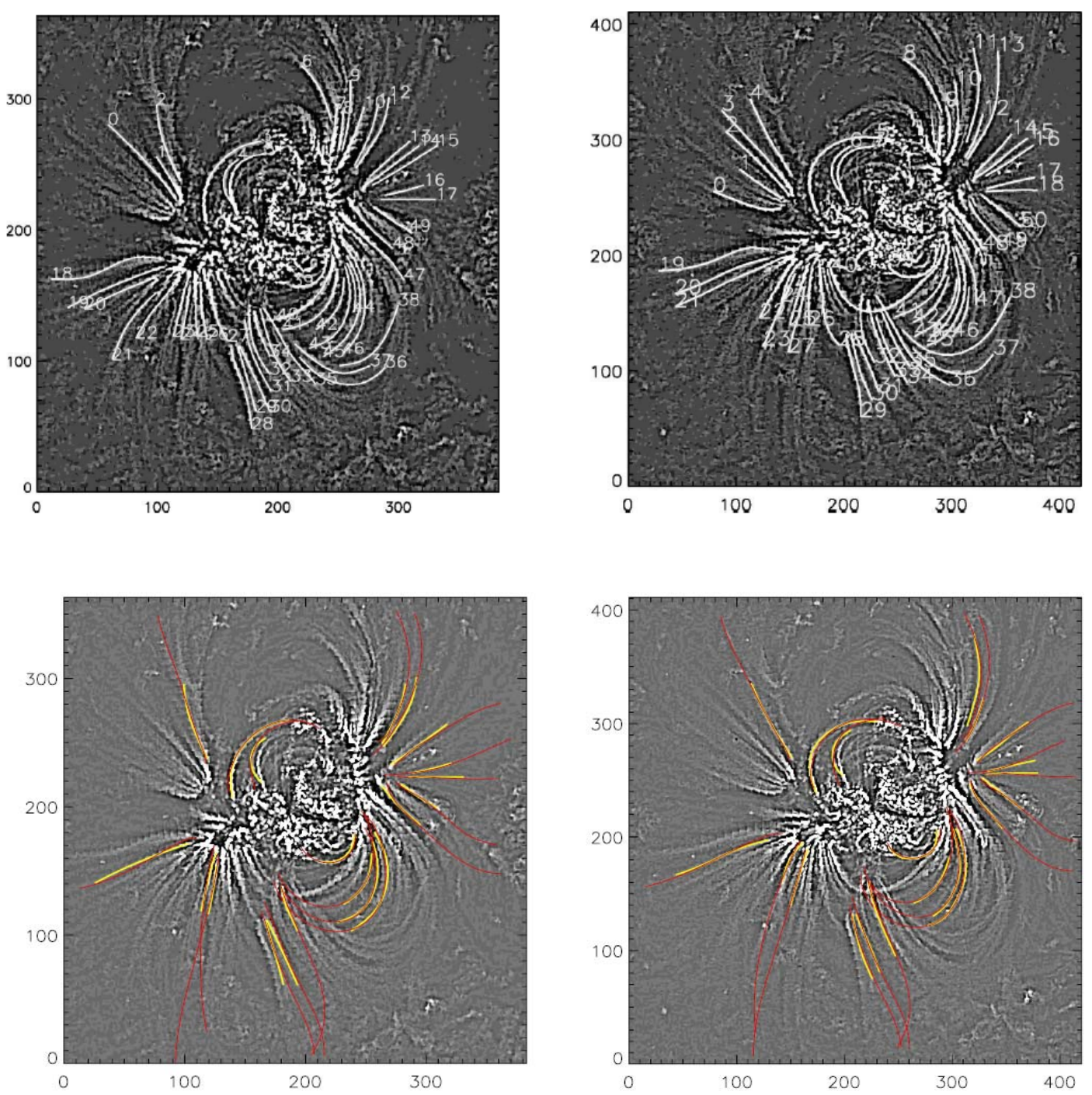

Fig. 2. Top: Contrast enhanced EUVI images from STEREO-B (left) and A (right) of the NOAA AR 0960 on 8 June 2007. The individual loops (enumerated white curves) have been extracted by a semi-automatic feature recognition tool as described in Inhester et al. (2008). Please note that equal numbers do not imply a correspondence across the images. Bottom: Some selected coronal loops (yellow) with their best fit magnetic field line (red). The separation angle between the spacecraft was $12^{\circ}$ (the top figures have been published originally in Feng et al., 2007b, Fig. 1).

feature recognition techniques is not satisfactory. The individual codes have control parameters, adapted to the image signal to noise ratio, resolution and also to the type of objects it aims to extract. A prime problem is that time-varying background loops and moss prevent an uncontaminated separation of loop and background. This makes it difficult to trace loop tops and footpoints. The problem becomes more complex for the comparison of EUV-images taken at different wavelengths and temperatures because the background is different in each filter. In the current stage the automatic feature recognition tools are already useful, but some human interaction is usually necessary and the codes can be considered as semi-automatic. The automated segmentation of polar coro- nal plumes, which we had during the recent solar minimum ample opportunity to observe (see Feng et al., 2009, for details), is more advanced. Even though they are fainter in intensity than loops, their shape is more restricted so that wavelet and the Hough transform can be employed for their detection. Llebaria et al. (2002) applied the Hough transform to a time series ( $66 \mathrm{~h}$ ) of SOHO/LASCO-C2 observations to generate time intensity diagrams of polar plumes. Such automated plume detection can be applied also to STEREOimages from two viewpoints (de Patoul et al., 2009). An alternative to stereoscopy is to study polar plumes with tomographic methods (see Barbey et al., 2008, for an application to SOHO/EIT images under the model assumption of "polar 
plumes as stationary objects whose intensity varies homogeneously with time."). Two STEREO-viewpoints are expected to be most useful for the tomography of plumes when the separation angle is about $60^{\circ}$.

\subsection{Association of objects in both images}

For faint objects like coronal EUV-loops it is not trivial to find associated structures in images taken from different vantage points as shown in Fig. 2. Rodriguez et al. (2009) developed a correlation tracking method which automatically matches pixels in both images, which worked well for small separation angles between spacecraft but it becomes difficult and ambiguous for if the separation angle of the spacecraft exceeds about $15^{\circ}$. In particular for large separation angles some structures might be visible in one image, but not in the other. Aschwanden et al. (2008c) applied a forward projection with an assumed height range of $h=0 \ldots 0.1 R_{S}$, which was sufficient to find correspondence for 30 traced loop, when the separation angle between STEREO-A and B was only $7^{\circ}$. The correspondence problem becomes more difficult to solve for larger separation angles.

Other possibilities are the use of a priori assumptions of the coronal structures, e.g., fitting to a semi-circular loop model (Aschwanden et al., 1999), or loop curvature constraints (Aschwanden, 2005). As an alternative one can use the fact that the emitting EUV-radiation outlines magnetic field lines due to the high conductivity of the coronal plasma. Consequently magnetic field lines should provide a reasonable proxy for coronal plasma loops. Wiegelmann and Neukirch (2002) used the stereoscopic reconstructed loops from Aschwanden et al. (1999) and photospheric magnetograms from SOHO/MDI to fit the optimum parameter $\alpha$ within the linear force-free field approximation. Projections of extrapolated 3-D magnetic field lines under different model assumptions have been compared with coronal images in Gary and Alexander (1999); Carcedo et al. (2003); Régnier and Amari (2004); Wiegelmann et al. (2005). The method has been extended for a STEREO pair of EUVimages in combination with linear and nonlinear force-free field models in Wiegelmann and Inhester (2006) and was dubbed magnetic stereoscopy. The idea of magnetic stereoscopy is that a number of 3-D field line proxies are projected onto the EUV-images and compared with the corresponding extracted curve-like structures (see Sect. 2.1). Loops in both images which have a minimum distance to the projection of a 3-D magnetic field line are very likely related to each other. The field line proxies where generated from extrapolation models but any other method to produce parameterized meaningful 3-D curves would work as well. The extrapolation models are here just a convenient means to generate 3-D curves the observed loops can be compared with. The method has been applied to TRACE-data (taken a day apart) by Feng et al. (2007b) and to STEREO/SECCHI by Feng et al. (2007a). In both cases the magnetic field has been com- puted with the linear force free method developed by Seehafer (1978) from SOHO/MDI. Force-free fields are characterized by $\nabla \times B=\alpha B$ and $B \cdot \nabla \alpha=0$, where $B$ is the magnetic field and $\alpha$ is zero for potential fields and constant in the entire space for linear force-free models. For more sophisticated non-linear force-free field models, $\alpha$ is constant along field lines but may vary on different field lines. An advantage of using coronal magnetic field models is that they generate meaningful and physics-based 3-D curves. Note, however, that a set of field lines from a linear force-free model constructed with a different $\alpha$ does not constitute a physically consistent magnetic field model. Disadvantages are that one needs additional observations from ground-based or spaceborne magnetographs (a third eye, e.g., SOHO or in future SDO) and that magnetic modelling and stereoscopy are not independent from each other, which is helpful for evaluating the consistency of both methods (see also discussion in Sect. 3).

\subsection{Geometric stereoscopy}

After having solved the feature extraction and association problem, the 3-D reconstruction is in principle only a geometric problem as indicated in Fig. 3 top panel. For stereoscopic reconstruction, it is helpful to introduce a suitable coordinate system - called "epipolar geometry" - which reduces the original 3-D reconstruction problem to a number of 2-D problems. The positions of the two STEREOspacecraft and any object point define a plane, called "epipolar plane", which serve as a natural coordinate system for the stereoscopic reconstruction (see Fig. 3 bottom panel). The projection of the epipolar planes onto the two STEREO image planes are called "epipolar lines". Per definition all epipolar lines in image-A converge at one point (marked as epipole of image 1 in Fig. 3 bottom panel) and vice versa. In practice the epipolar lines appear almost (but of course not strictly) parallel in images from the STEREO-EUVI (see Inhester, 2006, for quantitative estimations). As the STEREOspacecraft are close to the ecliptic the epipolar lines intersect with the rotation axis of the Sun (indicated as the z-axis in Fig. 3 bottom panel) and the angle with this axis can be used to label the epipolar lines. All points in space, except points on the stereo base line (the line which connects both spacecraft), are lying on a uniquely defined epipolar line. This is a helpful constraint as all points, visible on a specific epipolar line in image-A must lie also on the same epipolar line in image-B. In particular, for large separation angles, there is no guaranty that a faint coronal structure identified at observerA will be also visible at observer-B. This so called "epipolar constraint" (Inhester, 2006) is very helpful because it reduces the 3-D-stereoscopic reconstruction to calculating the position of each object on it's uniquely defined 2-D epipolar plane. The difference of the positions of an object projected along the epipolar lines in the two STEREO-images yields the depth information or in case of coronal loops the 

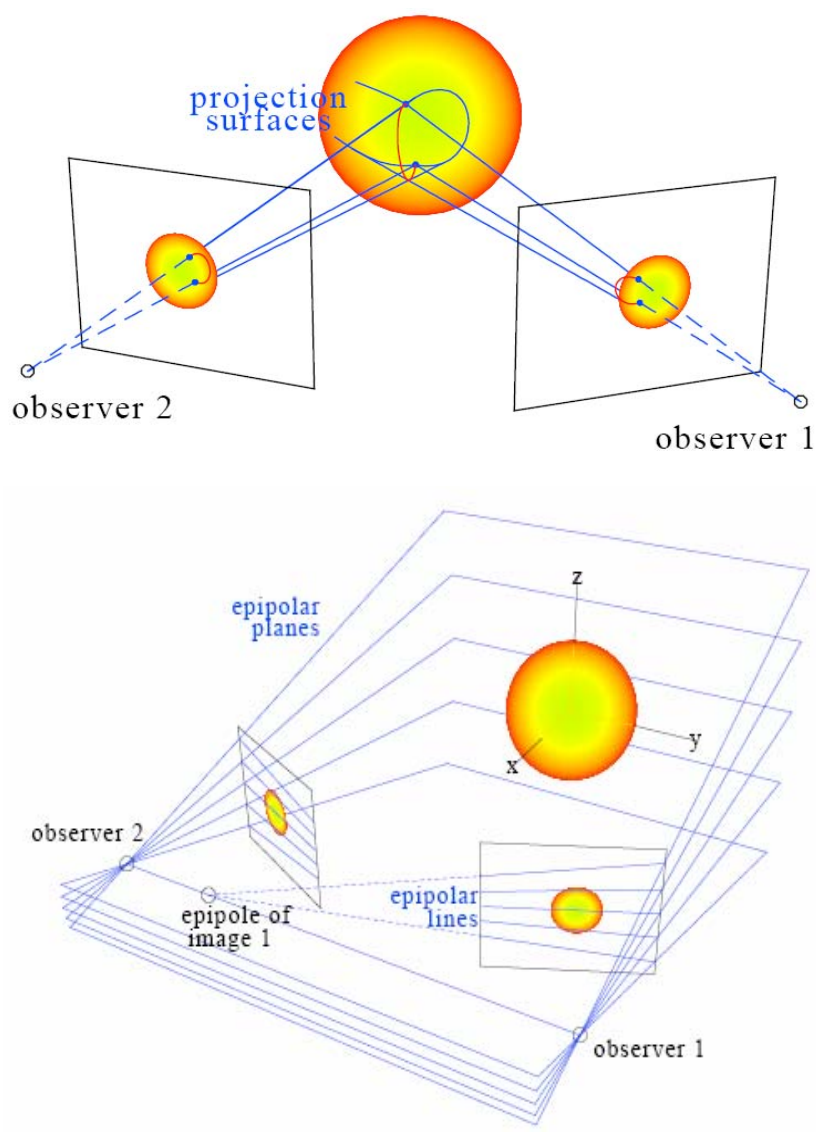

Fig. 3. Top panel: Back projection to reconstruct curve-like objects, e.g., coronal loops, from two images. Bottom panel: Epipolar planes and the corresponding epipolar lines in two STEREO-images (top and bottom panel of this figure have been originally published in Inhester, 2006, Figs. 1 and 2, respectively).

height in the corona. A simple way to tie-point associated loop curves in image A and B is to label along each curve the intersections with given epipolar lines and to reconstruct the intersection points with the same epipoles line label. This, of course, requires that both curves cover more or less the same epipolar range, a criterion which can be used to confirm a correct association.

\subsection{Estimating the reconstruction error in 3-D}

Features in the two STEREO-images can be identified of course only within a certain error margin as indicated by $w$ in Fig. 4 top panel. Possible error sources are the finite resolution of the instrument as well as uncertainties occurring due to extracting features from the EUV-images. The question is how do these uncertainties in the 2-D-images affect the reconstruction error of the 3-D coronal loop? As a consequence of the finite resolution $w$ of the loop projection, the true 3-D coordinate of a loop point lies nearby
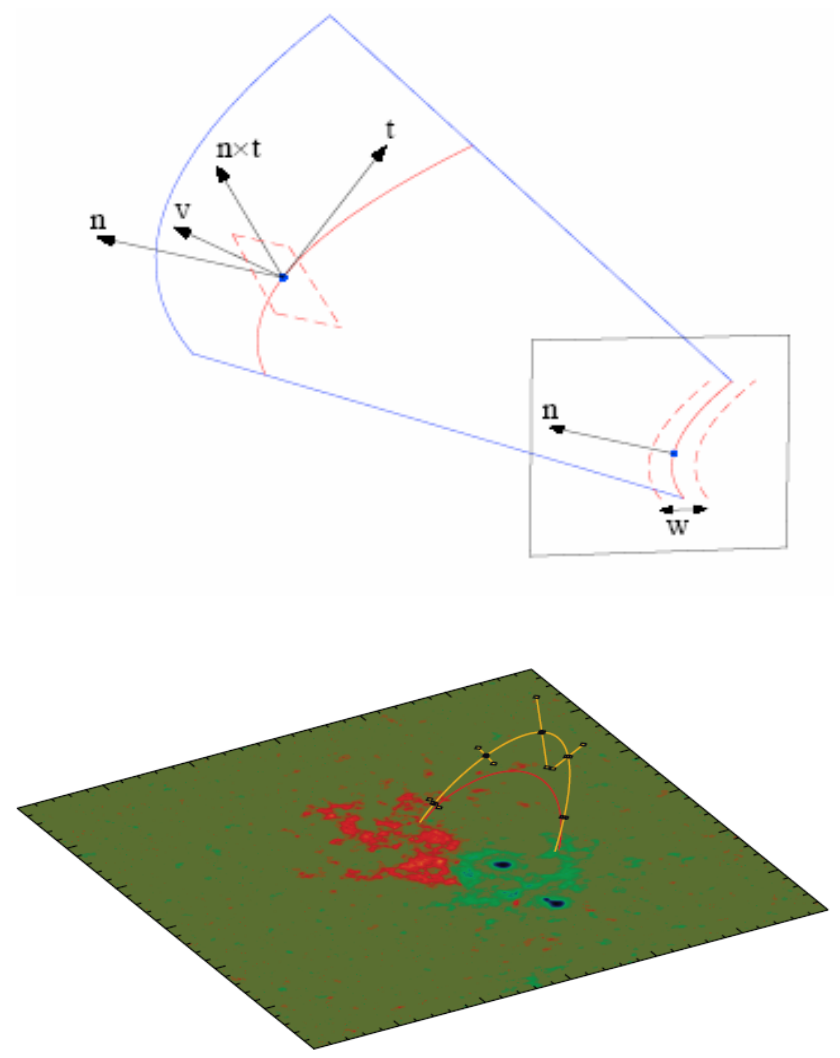

Fig. 4. Top: How does the uncertainty $w$ of the projected loop in both EUV-images affect the error-trapezoid of the reconstructed 3-D-loop? (see text) (original publishes in Inhester, 2006, Fig. 10), Bottom: Stereoscopic reconstructed 3-D loop (yellow) and best fit linear force free coronal magnetic field line extrapolated from SOHO/MDI magnetograms (original published in Feng et al., 2007a, Fig. 5).

in a plane through the reconstructed point which is spanned by the local normals $n_{i}$ of the two projection surfaces. $t=$ $\left(n_{1} \times n_{2}\right) /\left|n_{1} \times n_{2}\right|$ is the local curve tangent. Decomposing the uncertainty vector into its components $n_{1}$ and $n_{2}$ yields an error trapezoid of the positional uncertainty has the axes $w /(2 \cos (\alpha / 2))$ and $w /(2 \sin (\alpha / 2))$, where $\alpha$ is the angle between the projection surface normals (see Inhester, 2006, for a mathematical derivation and more details). For small $\alpha$ the depth error along the mean view direction of the two spacecraft may be considerable: $1 /(2 \sin (\alpha / 2))$ exceeds 5 for $\alpha<10^{\circ}$. $\alpha$ is limited from above by the angle between the two STEREO-spacecraft. This upper limit is reached when the loop intersects an epipolar line normally. This case allows the most accurate 3-D reconstruction for a given separation angle between spacecraft. For features parallel to epipolar lines $\alpha$ is zero and the 3-D reconstruction error becomes infinite. As epipolar lines are almost horizontal in the images this means that the highest 3-D reconstruction error occurs at the top of east-west loops. As a consequence, there are large 


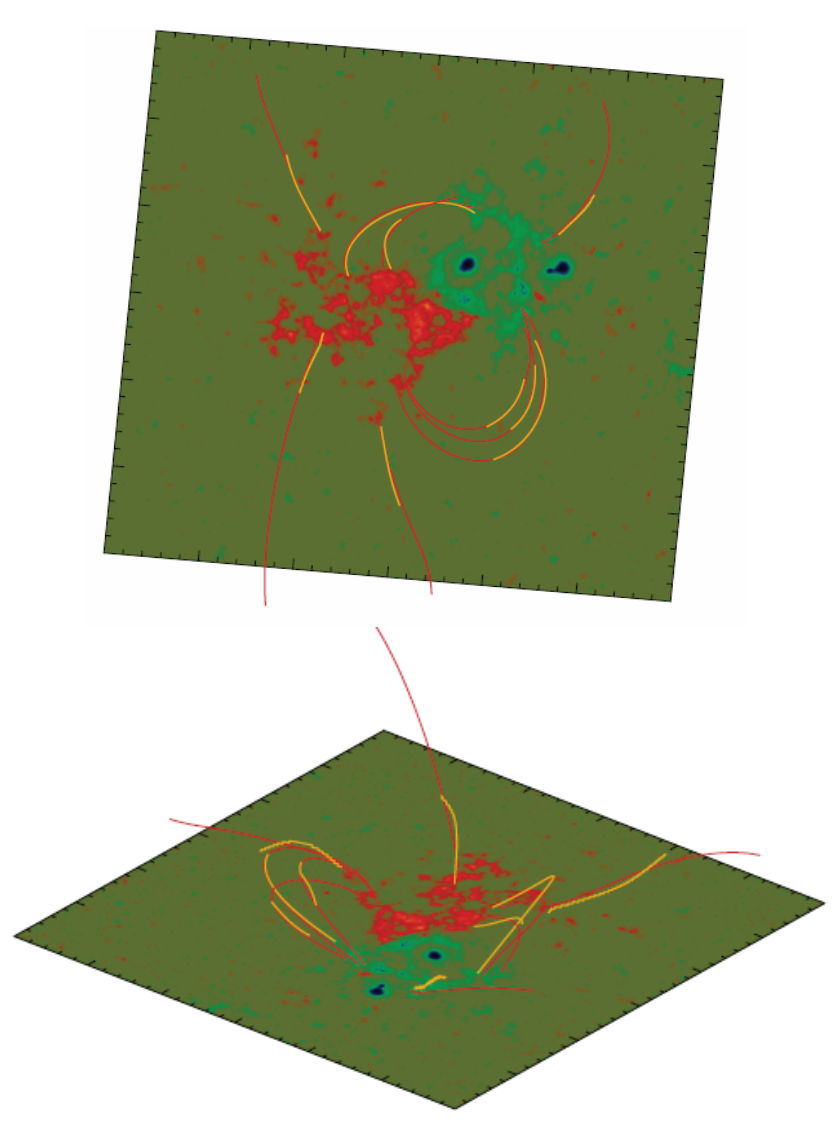

Fig. 5. Stereoscopic reconstructed 3-D loops and sections of loops (yellow) and best fit linear force-free field lines (red) from different viewpoints (top panel: view from STEREO-A, bottom panel: Northeast of the Active Region). (Original published in Feng et al., 2007a, Figs. 3 and 4.)

error bars in Fig. 4 bottom panel, which shown a stereoscopic reconstruction of one loop from (Feng et al., 2007a) for a separation angle of $12^{\circ}$ between spacecraft. A small (large) separation angle between spacecraft makes it easy (difficult) to associate related structures in both images, but for the 3-D reconstruction error it is the other way around, a small separation angle leads to a large error in 3-D.

An alternative error estimate has been given by Aschwanden et al. (2008c) for the uncertainty in height. Both error estimates show a similar behaviour, however: The smallest error is obtained if the loop tangent is normal to the epipolar planes and the error becomes infinite if the loop tangent becomes parallel to the epipolar plane.

Another problem in finding a unique solution for the 3D loop is a possible reconstruction ambiguity. This is a problem which can theoretically occur even for a pair of correctly identified loop projections, if the footpoints are wrongly associated in the image pair. In EUV images, the footpoints of loops are sometimes difficult to locate. They may be drowned in bundles of other loops or near-surface
EUV moss. This problem does, however, disappear if one can identify the footpoints of loops and requires them to be located near the solar surface. It is, however, not always possible to identify the loop footpoints and in this case one has two possible candidates for the true 3-D-solution. The true and the false reconstruction intersect at a point, where the projected segment is parallel to an epipolar line, e.g., the top of east-west coronal loops where the above error estimate formally diverges.

How can ambiguities and errors be limited? One possibility would be to use additional EUV-images from a third viewpoint, e.g., SOHO/EIT or in future SDO/AIA. This possibility has (to our knowledge) not been tried out yet. A potential problem might be the different resolution of the STEREO/SECCHI-EUVI and the SOHO/EUV instruments. The error in line-tying is also reduced by making use of the fact that the reconstructed loops should resemble field lines and hence should be smooth. Smoothing and/or spline fitting of the tie point reconstruction should in general reduce the reconstruction error.

Another possibility, which has been already shown to be useful for the association problem (Sect. 2.2), is to use coronal magnetic field models. This possibility, called "magnetic stereoscopy" was first tested with a model active region in Wiegelmann and Inhester (2006). Different magnetic field models, potential, linear and non-linear force-free have been used and it was shown that even the use of a poor field model (potential fields) was sufficient to resolve the reconstruction ambiguity. The extrapolated magnetic field lines provide already a proxy for the 3-D plasma loop and if ambiguous solutions occur, the solution closer to this proxy-loop is chosen. Figure 4 bottom panel and Fig. 5 shows a linear force-free field line (extrapolated from SOHO/MDI) in red, which has been used for this aim in Feng et al. (2007a). Extrapolated field lines might also serve as a reasonable approximation of the plasma loop in regions with a large reconstruction error, e.g., the loop top or if only parts of the loop are visible in the EUV-images.

\subsection{Derive physical quantities}

The geometry of the 3-D coronal structures, as seen from different viewpoints in Fig. 5 in yellow provide already useful information. Feng et al. (2007a) found that most of the reconstructed 3-D loops cannot be approximated by planar curve segments and that most of the loops are not circular. This was already known from field modelling. E.g. meaningful loop emissions per unit loop length can only be derived from EUV images, if the angle between the viewing direction and the loop tangent is properly taken into account (Aschwanden et al., 2008b).

In the following we will concentrate on the computation of physical plasma parameters, in particular the electron temperature and density along the reconstructed 3-D loops. Figure 6 shows the projection of a 3-D loop as reconstructed in 


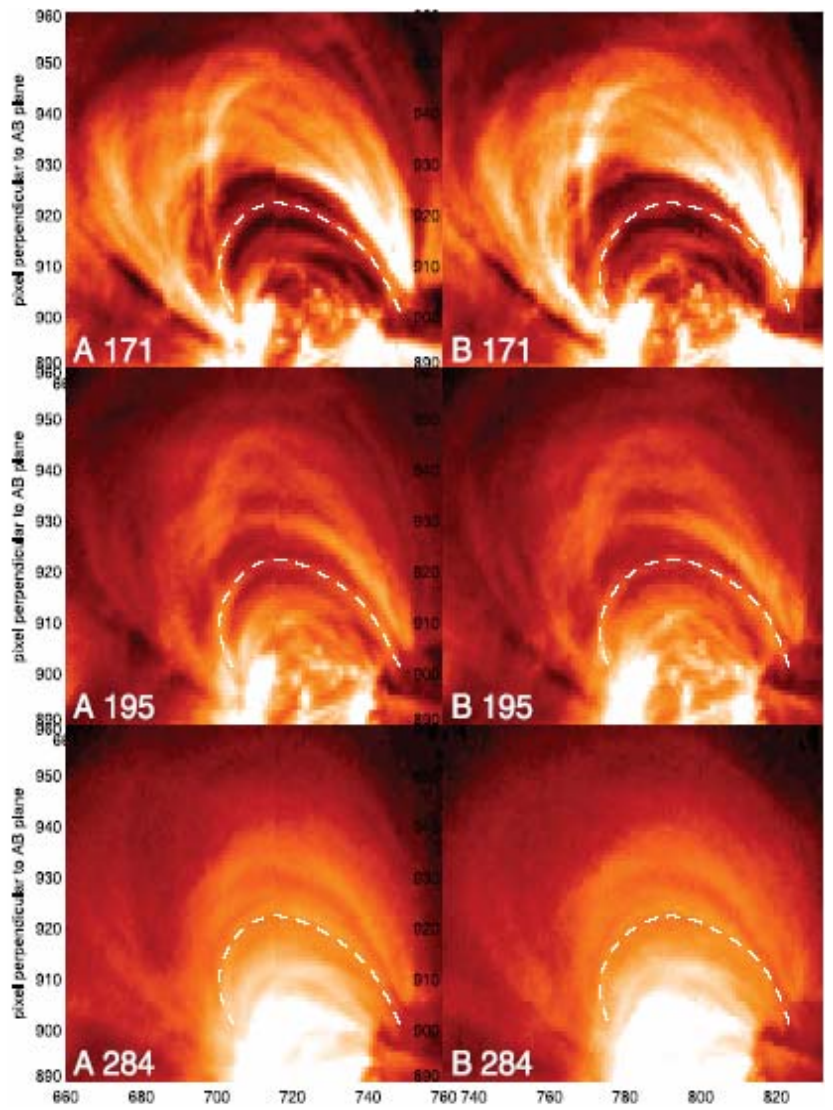

Fig. 6. EUVI-images at different wavelengths from STEREO-A and $\mathrm{B}$ in the left and right panels, respectively. The dashes white line shows a projections of a 3-D loop as reconstructed in (Aschwanden et al., 2008c). (Original published in Aschwanden et al., 2008b, Fig. 1).

(Aschwanden et al., 2008c) onto STEREO/SECCHI images at different wavelengths taken from STEREO-A and B in the left and right panels, respectively. As explained in detail in (Aschwanden et al., 2008b) the EUVI-images can be used to obtain the electron temperature and density, independent of each other for the EUVI-images taken from both STEREOviewpoints, which have been separated by $7^{\circ}$ for this study. As only a fraction of about $10 \%$ of the EUV-radiation is coming from the loops, one has to remove first the background separately in each wavelength, which is a tricky busyness and requires model assumptions as explained in detail in (Aschwanden et al., 2008b). After the well known temperature response functions (defined for each wavelength), as calculated with the CHIANTI-code, are used to obtain physical quantities along the loops. For this aim a local loop-aligned coordinate system is introduced and the differential emission measure (DEM) is constrained for each loop position with 3 temperature filters at various positions along the loop with a Gaussian function. The DEM is then fitted with the help of the EUVI response functions in three wavelength, which pro-

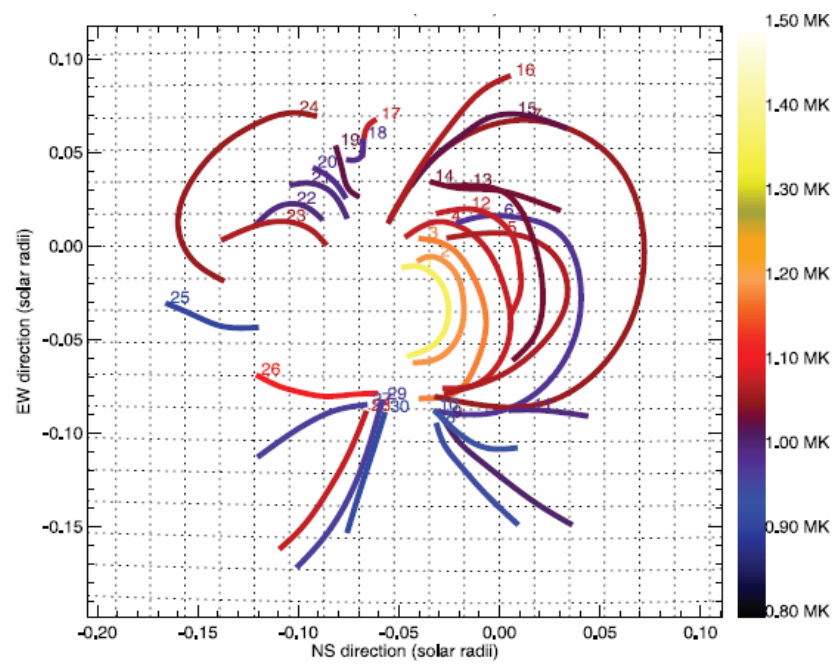

Fig. 7. Temperature map of 30 loops. The loops are isothermal within the error estimation and the hottest loops are also the smallest. (Original published in Aschwanden et al., 2008b, as part of Fig. 9).

vides approximations for the temperature, the Gaussian half width of DEM and the peak emission measure at the chosen positions along the loop (see Aschwanden et al., 2008b, Eqs. 1-4 for details). Figure 7 shows the temperature for 30 plasma loops. The loop-temperatures computed with this technique where found isothermal along the loop (within an error margin of about $20 \%$ caused mainly by background substraction uncertainties). The temperature scales with loop length with the shortest loops are also the hottest ones. For a known loop width (as shown in Fig. 4, upper panel) the three parameters of the DEM-model (temperature, Gaussian half width, peak emission measure) can be converted into electron densities (see Aschwanden et al., 2008b, Sect. 3.4, Eqs. 5-10 for details). With the stereoscopic reconstructed 3-D-loop projected onto images from STEREO-A and B the temperature and density can be calculated independently from both spacecraft. There have been some systematic differences regarding the results from both spacecraft, e.g., the average loop temperature computed from STEREO-B was somewhat higher as the one from STEREO-A, but the discrepancies have been only a few percent for density and temperature estimations. It is still to be investigated how consistent the estimations from both spacecraft are for larger separation angles. Coronal stereoscopy provides us for the first time with the 3-D-loop length, temperature and density. These quantities can be used to test RTV-scaling laws (Rosner et al., 1978) and to go a further step towards a self-consistent modelling of the solar corona, which will be discussed in Sect. 4 . 


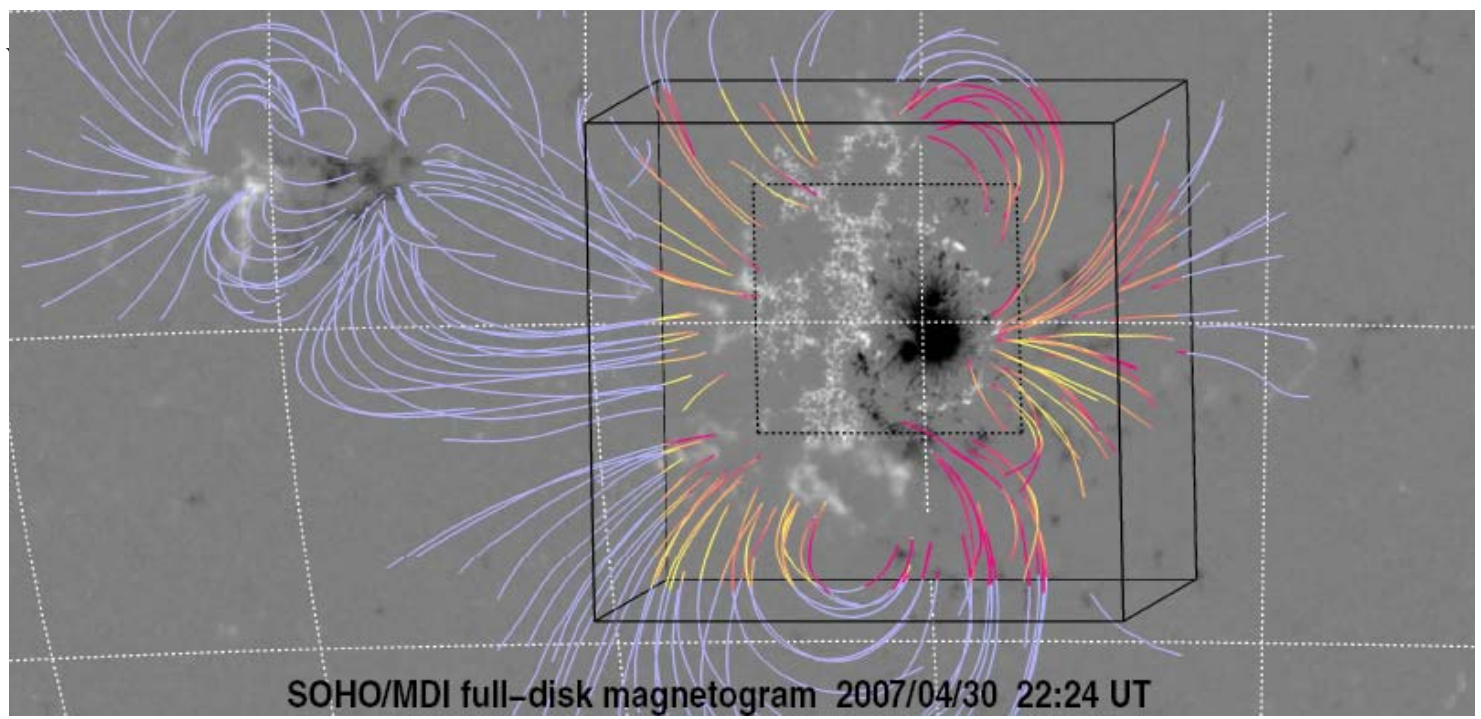

Fig. 8. SOHO/MDI magnetogram of AR 10953 with over-plotted stereoscopic reconstructed loops (see Aschwanden et al., 2008c) in blue and extrapolated non-linear force-free coronal magnetic field lines. The solid box depicts the $320 \times 320 \times 256$ grid point simulation box in which the magnetic field extrapolations have been carried out. The dotted area depicts the field-of-view of Hinode/SOT-SP. Only in this dotted area photospheric vector magnetograms have been available. (Original published in DeRosa et al., 2009, as part of Fig. 1).

\section{Stereoscopy and coronal modelling}

As explained in the previous section, coronal magnetic field models provide useful information for solving the stereoscopic correspondence problem (see Sect. 2.2) and to remove reconstruction ambiguities (Sect. 2.4). Until now mainly linear force-free models have been used for this aim and the method automatically fits the optimum linear force-free parameter $\alpha$ for each loop individually to approximate its shape as closely as possible. $\alpha$ is here just used as a numerical parameter to alter the curve shape. It is therefore not surprising that Feng et al. (2007a) found a significant scatter of $\alpha$ between the loops. We cannot interpret the values of $\alpha$ in terms of a linear force-free magnetic field model, because the different values are a contradiction to the premises of a globally constant $\alpha$. For a physical meaningful self-consistent magnetic field model one cannot determine the values of $\alpha$ independently for each loop. Unfortunately, a physical consistent nonlinear model is way more demanding, both computationally due to the intrinsic nonlinearity of the model and from an observational point of view since nonlinear models require photospheric vector magnetograms as input. Within the last few years a group of scientists (nonlinear force-free field consortium, chaired by C. Schrijver) has intensively compared and evaluated corresponding computer codes (Schrijver et al., 2006, 2008; Metcalf et al., 2008), which showed the codes produce reliable results, when feeded with consistent input data (vector magnetograms or a quantity derived from vector magnetograms). In another joint study of the consortium (by DeRosa et al., 2009) the codes have been applied to AR 10953 and the 3-D structure of the magnetic field lines has been compared with the 3-D geometry of plasma loops (as stereoscopically reconstructed in Aschwanden et al., 2008c). Figure 8 shows the stereo-loops in blue and in red and yellow the magnetic field lines. A major difficulty of this study was that the Hinode-SOT/SP vector magnetograms, required as input for the magnetic field codes, where available in only a very small field of view (dotted area in Fig. 8) and has only about $10 \%$ of the area spanned by the stereo loops. The majority of stereo-reconstructed loops were located outside of this region, therefore the reconstruction box was significantly enlarged beyond the Hinode-area (solid lines in Fig. 8, but only the line of sight component of the photospheric magnetic field from SOHO/MDI was available in this enlarged box. Assumptions regarding the transverse photospheric field component had to be made in the MDI-area, but unfortunately the various magnetic field codes made different use of the assumption in the MDI-area and consequently the resulting magnetic field lines differed between the codes. The extrapolated magnetic field lines turned out to be also inconsistent with the reconstructed STEREOloops. There was an average misalignment angle of $24^{\circ}$ between field lines and loops. Sandman et al. (2009) compared stereoscopic reconstructed loops for three active regions observed in April and May 2007 with potential field extrapolations and found a misalignment angle of about $20^{\circ}-40^{\circ}$. In the DeRosa et al. (2009) study the nonlinear force-free approach did not provide better agreement with the stereoloops than the much simpler to compute potential fields. A main reason seems to be the very small field of view, where 
actually vector magnetogram data have been available and correspondingly the codes have not been fed with a consistent input. As visible in Fig. 8 almost no field line/STEREOloop closes within the Hinode field of view. It is therefore necessary to repeat such a study with much larger field of view of vector magnetogram data (ideally at least the same FOV as spanned by the STEREO-loops).

An additional complication is that the magnetic field vector is measured routinely only in the photosphere, where the magnetic field is not force-free due to the high plasma $\beta$ (see Metcalf et al., 1995). Consequently photospheric vector magnetograms do not provide consistent boundary conditions for a nonlinear force-free extrapolation. To overcome this difficulty, a preprocessing method has been developed to remove the non-magnetic forces from the photospheric vector magnetograms (see Wiegelmann et al., 2006; Fuhrmann et al., 2007, for details). These preprocessed magnetograms are more chromospheric like and chromospheric observations, e.g., can be incorporated into the preprocessing-algorithm as described in Wiegelmann et al. (2008). In principle one could incorporate additional observational constraints into the preprocessing routine, for example minimize the angle of stereoscopic reconstructed loops (at the footpoints) with the magnetic field vector. This might help to better estimate the magnetic field vector in the upper chromosphere. Another possibility which might be tried out is to add a term which minimizes the angle between the coronal magnetic field and the reconstructed 3-D coronal loops in the nonlinear force-free modelling algorithm. One possibility would be to extend the nonlinear force-free optimization principle Wheatland et al. (2000); Wiegelmann (2004) by means of a Lagrangian multiplier $\zeta$ as suggested in Eq. (1).

$$
\begin{aligned}
L= & \int_{V}\left[B^{-2}|(\nabla \times \mathbf{B}) \times \mathbf{B}|^{2}+|\nabla \cdot \mathbf{B}|^{2}\right] d^{3} V+ \\
& \zeta \int_{V}\left(\mathbf{B} \times \mathbf{S}_{3 \mathrm{D}}\right)^{2} d^{3} V
\end{aligned}
$$

The first two terms correspond to the force-free equations and the last term measures the angle between the coronal magnetic field $\mathbf{B}$ and the stereoscopic reconstructed 3-Dloops $\mathbf{S}_{3 \mathrm{D}}$, where $\mathbf{S}_{3 \mathrm{D}}$ should contain also an error approximation of the stereoscopic reconstruction error This could be done by the local length of the vector $\mathbf{S}_{3 \mathrm{D}}$ along the stereo-loop, where $\left|\mathbf{S}_{3 \mathrm{D}}\right|=1$ would indicate a small error and $\left|\mathbf{S}_{3 \mathrm{D}}\right|=0$ an infinite error. Locations with $\left|\mathbf{S}_{3 \mathrm{D}}\right|=0 \mathrm{ob}-$ viously do not contribute to the functional. The third term in Eq. (1) corresponds to a weighted angle between magnetic field and STEREO-loops. Regions with high magnetic field strength and accurate measurement of $\left|\mathbf{S}_{3 \mathrm{D}}\right|$ contribute more to the functional. As such data (an active region observed simultaneously by both STEREO-spacecraft and a large field-of-view vector magnetograph) seem not to be currently available (also due to a lack of active regions in recent years), corresponding studies have to be postponed until after the launch of SDO. The SDO/HMI intrument will pro- vide full disc vectormagnetograph, which resolves the limited FOV-problem of current instruments. However, the angle between the spacecraft might then be too large for stereoscopy with STEREO A/B spacecraft. One has to evaluate whether SDO/AIA and one of the STEREO-spacecraft can be used for stereoscopy instead. The result can then be compared with nonlinear force-free extrapolations from SDO/HMI. This instrument will provide the required larger FOV for the field modelling.

\section{Where to go in coronal stereoscopy?}

To summarize the current state of the art of coronal stereoscopy we propose a concept of five steps, namely: feature extraction, association, geometric reconstruction, error approximation and physical modelling. Several improvements are still possible for some of the steps, e.g., a fully automatic and reliable feature recognition method, investigations on how stereoscopy is still possible with larger separation angles between the spacecraft and if we can combine also STEREO with other missions, e.g., SDO. Some basic difficulties, e.g., the large reconstruction error on the top of eastwest loop is an intrinsic problem of stereoscopy, unless we have one or more spacecraft well above or below the ecliptic.

Despite these principal difficulties coronal stereoscopy provides us for the first time some information about the 3-D geometry and physical quantities of plasma loops. A useful concept has been also to combine stereoscopy with magnetic modelling of the solar corona, but due to shortage of vector magnetogram data these approaches have been mainly done with linear force-free methods. With the forthcoming full disc vector magnetograph SDO/HMI nonlinear forcefree magnetic field models are assumed to become available on a regular basis. A principal problem of force-free magnetic field modelling is that it does not include a selfconsistent modelling of the coronal plasma. Assume a static corona model for example, which balances the Lorentz-force with pressure gradient and gravity as $j \times B=\nabla p+\rho \nabla \psi$. The term force-free means that the Lorentz force vanishes and correspondingly the current density $j$ is parallel to the magnetic field $B$. While this approach is well justified for the field modelling in the low plasma $\beta$ solar corona, it means also that $\nabla p+\rho \nabla \psi=0$, and because the gravity force $-\nabla \psi$ is in the radial direction a consequence is that the plasma would only be gravitationally stratified, contrary to observations. Consequently we cannot describe the solar coronal plasma self-consistently within a force-free model. Due to the low coronal plasma $\beta$ the force-free assumption is well justified to compute the coronal magnetic field structure. Variations in the plasma pressure along the field lines are then compensated by relatively small Lorentz-forces. For a self-consistent modelling of the solar corona we have to take this effect into account and use a magneto-hydro-static model in lowest order. 


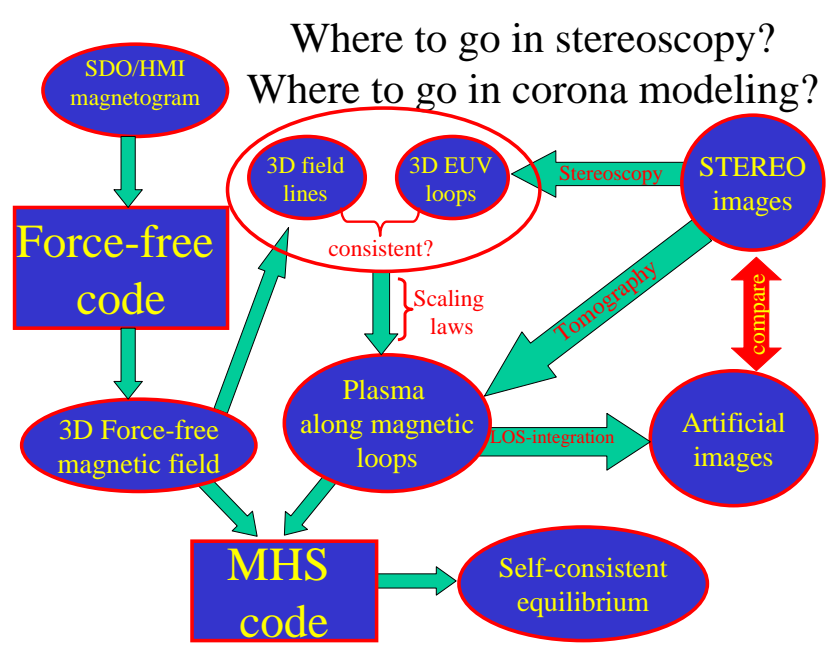

Fig. 9. A concept on how stereoscopy could be imbedded in a selfconsistent coronal modelling approach.

In Fig. 9 we outline an approach towards a self-consistent coronal modelling. First there should be two alternative routes to derive the 3-D geometry of coronal loops, either from two EUV-images by stereoscopy or by force-free extrapolations from vector magnetograms, e.g., as provided in future from SDO/HMI. The resulting 3-D field lines and 3-D EUV-loops should be consistent with each other. Unfortunately this has not been the case for a first comparison (by DeRosa et al., 2009) as discussed already in Sect. 3, but it is assumed (or at least hoped for)that the new instrumentation with SDO/HMI and improvements in modelling will lead to more consistent results. If consistency between plasma loops and field lines has been found, we will have a much more reliable magnetic field model than we obtain from extrapolation alone. One can then derive physical quantities along the loops as outlined in Sect. 2.5. An alternative method is to use a tomographic approach (as explained in detail in Aschwanden et al., 2009), which provides the plasma density without model assumptions. A sophisticated modelling of quantities along the loops can be done (see Schrijver et al., 2004, who used scaling laws between the photospheric magnetic field and the heating rate at the footpoints of loops). From physical quantities like density and temperature one can compute artificial EUV-images and compare them with the real STEREO-images. This approach might as well allow to adjust free parameters within the loop modelling approach. As pointed out above, modelling of coronal magnetic field and plasma cannot be achieved a selfconsistently with a force-free model. Plasma loop modelling will create small gravity and plasma pressure forces, which have to be compensated by a Lorentz-forces. Due to the low coronal plasma $\beta$, the Lorentz force required will be very small and the coronal magnetic field structure will deviate only marginally from a force-free model.
Finally a self-consistent model using the magneto-hydrostatic approach can be computed with the force-free magnetic field model and the plasma along the loops as input. Corresponding magneto-hydro-static codes have been developed and tested in Wiegelmann and Neukirch (2006); Wiegelmann et al. (2007) in cartesian and spherical geometry, respectively. The resulting consistent static model can be used as input to investigate dynamic phenomena for example with time-dependent MHD-codes.

With stereoscopically reconstructed loops, even if they are few in number, we will have for the first time an indirect though quantitative measurement of the local coronal magnetic field direction. In future magnetic field extrapolation codes, this information should be used not only for a comparison but as an additional constraint to lift some of the uncertainties which arise from unknown boundary values. For a forecast of the evolution and stability of observed active regions, we probably will need time dependent simulations of the corona which incorporate observations by data assimilation schemes similar to the way they are now used in computational meteorology (see Daley, 1991). An anfolding of the solar remote sensing observations to constrain the 3-D state of the solar corona is vital for these schemes and the forecast range will strongly depend on the quality of the 3-D reconstructions. For this task, the techniques for detecting well defined objects in the images and the way they are processed into 3-D structures needs to be improved. The present state of the art of solar stereoscopy can only be a first step in this direction.

A challenging task in stereoscopy is the 3-D reconstruction of true dynamic phenomena like the initiation of flares and CMEs. A key question is which role magnetic reconnection plays for such eruptive phenomena. Stereoscopy could help us first to derive the quasi-stationary state before an eruption, preferably within a sophisticated self-consistent model, e.g., magneto-hydro-statics. If we can then also observe the 3-Dstructure of the dynamic phase this would be very helpful for our understanding of such phenomena. One could, in particular, use the self-consistent stationary state as input for time dependent simulations, e.g., with MHD or Hall-MHD. It is notoriously difficult to find transport coefficients, e.g., resistivity, viscosity, heat tensor etc. for the coronal plasma from micro-physics for the coronal plasma, because the required kinetic scales are way to small to observe. One could, however, try to optimize these transport coefficient with a systematic trial and error approach in order to fit the observations best. Such observational based simulations would provide us then a rich new world of insights. If the dynamic model simulations show reasonable agreement with observed quantities, we might also have some confidence in other model quantities, which cannot be observed. This could give insights about the physics of magnetic reconnection in the coronal plasma.

A good knowledge of the physics of the solar corona, say the 3-D structure of loops and plasma along the loops will 
be very helpful, together with high accuracy measurements of the photospheric magnetic field vector, to understand the interface region between photosphere and corona. A physical understanding of this region is important for the coronal heating problem. Coronal stereoscopy can help here because it provides a fair approximation of the corona, but additional direct observations of the interface region, as provided in near future for example from the small explorer mission IRIS are necessary. A modelling approach in this region is very challenging, because low and high $\beta$ plasma with suband supersonic plasma flows exist side by side here.

Acknowledgements. We would like to thank the organization committee (chaired by Richard Harrison) of the STEREO-3/SOHO-22 workshop "Three eyes on the Sun" for the invitation to give this review on stereoscopy. This work was supported by DLR-grant 50 OC 0501. The SECCHI data used here were produced by an international consortium of the Naval Research Laboratory (USA), Lockheed Martin Solar and Astrophysics Lab (USA), NASA Goddard Space Flight Center (USA), Rutherford Appleton Laboratory (UK), University of Birmingham (UK), Max-Planck-Institut for Solar System Research (Germany), Centre Spatiale de Liège (Belgium), Institut d'Optique Thèorique et Appliquèe (France), and Institut d'Astrophysique Spatiale (France).

The service charges for this open access publication have been covered by the Max Planck Society.

Topical Editor R. Forsyth thanks two anonymous referees for their help in evaluating this paper.

\section{References}

Aschwanden, M. J.: 2D Feature Recognition And 3d Reconstruction In Solar Euv Images, Sol. Phys., 228, 339-358, doi:10.1007/ s11207-005-2788-5, 2005.

Aschwanden, M. J., Newmark, J. S., Delaboudinière, J.-P., Neupert, W. M., Klimchuk, J. A., Gary, G. A., Portier-Fozzani, F., and Zucker, A.: Three-dimensional Stereoscopic Analysis of Solar Active Region Loops. I. SOHO/EIT Observations at Temperatures of (1.0-1.5) X 10^6 K, ApJ, 515, 842-867, doi: 10.1086/307036, 1999.

Aschwanden, M. J., Alexander, D., Hurlburt, N., Newmark, J. S., Neupert, W. M., Klimchuk, J. A., and Gary, G. A.: Three-dimensional Stereoscopic Analysis of Solar Active Region Loops. II. SOHO/EIT Observations at Temperatures of 1.5-2.5 MK, ApJ, 531, 1129-1149, doi:10.1086/308483, 2000.

Aschwanden, M. J., Lee, J. K., Gary, G. A., Smith, M., and Inhester, B.: Comparison of Five Numerical Codes for Automated Tracing of Coronal Loops, Sol. Phys., 248, 359-377, doi: 10.1007/s11207-007-9064-9, 2008a.

Aschwanden, M. J., Nitta, N. V., Wuelser, J.-P., and Lemen, J. R.: First 3D Reconstructions of Coronal Loops with the STEREO A+B Spacecraft. II. Electron Density and Temperature Measurements, ApJ, 680, 1477-1495, doi:10.1086/588014, 2008b.

Aschwanden, M. J., Wülser, J.-P., Nitta, N. V., and Lemen, J. R.: First Three-Dimensional Reconstructions of Coronal Loops with the STEREO A and B Spacecraft. I. Geometry, ApJ, 679, 827842, doi:10.1086/529542, 2008 c.
Aschwanden, M. J., Wuelser, J.-P., Nitta, N. V., Lemen, J. R., and Sandman, A.: First Three-Dimensional Reconstructions of Coronal Loops with the STEREO A+B Spacecraft. III. Instant Stereoscopic Tomography of Active Regions, ApJ, 695, 12-29, doi: 10.1088/0004-637X/695/1/12, 2009.

Barbey, N., Auchère, F., Rodet, T., and Vial, J.-C.: A TimeEvolving 3D Method Dedicated to the Reconstruction of Solar Plumes and Results Using Extreme Ultraviolet Data, Sol. Phys., 248, 409-423, doi:10.1007/s11207-008-9151-6, 2008.

Batchelor, D.: Quasi-stereoscopic imaging of the solar X-ray corona, Sol. Phys., 155, 57-61, 1994.

Berton, R. and Sakurai, T.: Stereoscopic determination of the threedimensional geometry of coronal magnetic loops, Sol. Phys., 96, 93-111, 1985.

Carcedo, L., Brown, D. S., Hood, A. W., Neukirch, T., and Wiegelmann, T.: A Quantitative Method to Optimise Magnetic Field Line Fitting of Observed Coronal Loops, Sol. Phys., 218, 29-40, 2003.

Daley, R.: Atmospheric Data Analysis, Cambridge Atmospheric and Space Science Series, 1991.

DeRosa, M. L., Schrijver, C. J., Barnes, G., Leka, K. D., Lites, B. W., Aschwanden, M. J., Amari, T., Canou, A., McTiernan, J. M., Régnier, S., Thalmann, J. K., Valori, G., Wheatland, M. S., Wiegelmann, T., Cheung, M. C. M., Conlon, P. A., Fuhrmann, M., Inhester, B., and Tadesse, T.: A Critical Assessment of Nonlinear Force-Free Field Modeling of the Solar Corona for Active Region 10953, ApJ, 696, 1780-1791, doi: 10.1088/0004-637X/696/2/1780, 2009.

Feng, L., Inhester, B., Solanki, S. K., Wiegelmann, T., Podlipnik, B., Howard, R. A., and Wuelser, J.-P.: First Stereoscopic Coronal Loop Reconstructions from STEREO SECCHI Images, ApJL, 671, L205-L208, doi:10.1086/525525, 2007a.

Feng, L., Wiegelmann, T., Inhester, B., Solanki, S., Gan, W. Q., and Ruan, P.: Magnetic Stereoscopy of Coronal Loops in NOAA 8891, Solar Phys., 241, 235-249, doi:10.1007/ s11207-007-0370-z, 2007b.

Feng, L., Inhester, B., Solanki, S. K., Wilhelm, K., Wiegelmann, T., Podlipnik, B., Howard, R. A., Plunkett, S. P., Wuelser, J. P., and Gan, W. Q.: Stereoscopic Polar Plume Reconstructions from STEREO/SECCHI Images, ApJ, 700, 292-301, doi: 10.1088/0004-637X/700/1/292, 2009.

Fuhrmann, M., Seehafer, N., and Valori, G.: Preprocessing of solar vector magnetograms for force-free magnetic field extrapolation, A\&A, 476, 349-357, doi:10.1051/0004-6361:20078454, 2007.

Gary, G. A. and Alexander, D.: Constructing the Coronal Magnetic Field By Correlating Parameterized Magnetic Field Lines With Observed Coronal Plasma Structures, Sol. Phys., 186, 123-139, 1999.

Howard, R. A., Moses, J. D., Vourlidas, A., Newmark, J. S., Socker, D. G., Plunkett, S. P., Korendyke, C. M., Cook, J. W., Hurley, A., Davila, J. M., Thompson, W. T., St Cyr, O. C., Mentzell, E., Mehalick, K., Lemen, J. R., Wuelser, J. P., Duncan, D. W., Tarbell, T. D., Wolfson, C. J., Moore, A., Harrison, R. A., Waltham, N. R., Lang, J., Davis, C. J., Eyles, C. J., Mapson-Menard, H., Simnett, G. M., Halain, J. P., Defise, J. M., Mazy, E., Rochus, P., Mercier, R., Ravet, M. F., Delmotte, F., Auchere, F., Delaboudiniere, J. P., Bothmer, V., Deutsch, W., Wang, D., Rich, N., Cooper, S., Stephens, V., Maahs, G., Baugh, R., McMullin, D., and Carter, T.: Sun Earth Connection Coronal and Helio- 
spheric Investigation (SECCHI), Space Sci. Rev., 136, 67-115, doi:10.1007/s11214-008-9341-4, 2008.

Inhester, B.: Stereoscopy basics for the STEREO mission, ArXiv Astrophysics e-prints, 2006.

Inhester, B., Feng, L., and Wiegelmann, T.: Segmentation of Loops from Coronal EUV Images, Solar Phys., 248, 379-393, doi:10. 1007/s11207-007-9027-1, 2008.

Kaiser, M. L., Kucera, T. A., Davila, J. M., St. Cyr, O. C., Guhathakurta, M., and Christian, E.: The STEREO Mission: An Introduction, Space Sci. Rev., 136, 5-16, doi:10.1007/ s11214-007-9277-0, 2008.

Llebaria, A., Thernisien, A., and Lamy, P.: Characterization of the polar plumes from high cadence LASCO-C2 observations, Adv. Space Res., 29, 343-348, doi:10.1016/S0273-1177(01)00595-6, 2002.

Metcalf, T. R., Jiao, L., McClymont, A. N., Canfield, R. C., and Uitenbroek, H.: Is the solar chromospheric magnetic field forcefree?, ApJ, 439, 474-481, doi:10.1086/175188, 1995.

Metcalf, T. R., Derosa, M. L., Schrijver, C. J., Barnes, G., van Ballegooijen, A. A., Wiegelmann, T., Wheatland, M. S., Valori, G., and McTiernan, J. M.: Nonlinear Force-Free Modeling of Coronal Magnetic Fields. II. Modeling a Filament Arcade and Simulated Chromospheric and Photospheric Vector Fields, Solar Phys., 247, 269-299, doi:10.1007/s11207-007-9110-7, 2008.

Régnier, S. and Amari, T.: 3D magnetic configuration of the $\mathrm{H} \alpha$ filament and X-ray sigmoid in NOAA AR 8151, A\&A, 425, 345352, 2004.

Rodriguez, L., Zhukov, A. N., Gissot, S., and Mierla, M.: ThreeDimensional Reconstruction of Active Regions, Solar Phys., 256, 41-55, doi:10.1007/s11207-009-9355-4, 2009.

Rosner, R., Tucker, W. H., and Vaiana, G. S.: Dynamics of the quiescent solar corona, ApJ, 220, 643-645, doi:10.1086/155949, 1978.

Sandman, A. W., Aschwanden, M. J., Derosa, M. L., Wülser, J. P., and Alexander, D.: Comparison of STEREO/EUVI Loops with Potential Magnetic Field Models, Solar Phys., published online first, 81, doi:10.1007/s11207-009-9383-0, 2009.

Schrijver, C. J., Title, A. M., Berger, T. E., Fletcher, L., Hurlburt, N. E., Nightingale, R. W., Shine, R. A., Tarbell, T. D., Wolfson, J., Golub, L., Bookbinder, J. A., Deluca, E. E., McMullen, R. A., Warren, H. P., Kankelborg, C. C., Handy, B. N., and de Pontieu, B.: A new view of the solar outer atmosphere by the Transition Region and Coronal Explorer, Solar Phys., 187, 261-302, 1999.

Schrijver, C. J., Sandman, A. W., Aschwanden, M. J., and DeRosa, M. L.: The Coronal Heating Mechanism as Identified by FullSun Visualizations, ApJ, 615, 512-525, doi:10.1086/424028, 2004.
Schrijver, C. J., Derosa, M. L., Metcalf, T. R., Liu, Y., McTiernan, J., Régnier, S., Valori, G., Wheatland, M. S., and Wiegelmann, T.: Nonlinear Force-Free Modeling of Coronal Magnetic Fields Part I: A Quantitative Comparison of Methods, Solar Phys., 235, 161-190, doi:10.1007/s11207-006-0068-7, 2006.

Schrijver, C. J., DeRosa, M. L., Metcalf, T., Barnes, G., Lites, B., Tarbell, T., McTiernan, J., Valori, G., Wiegelmann, T., Wheatland, M. S., Amari, T., Aulanier, G., Démoulin, P., Fuhrmann, M., Kusano, K., Régnier, S., and Thalmann, J. K.: Nonlinear Force-free Field Modeling of a Solar Active Region around the Time of a Major Flare and Coronal Mass Ejection, ApJ, 675, 1637-1644, doi:10.1086/527413, 2008.

Seehafer, N.: Determination of constant alpha force-free solar magnetic fields from magnetograph data, Solar Phys., 58, 215-223, 1978.

Wheatland, M. S., Sturrock, P. A., and Roumeliotis, G.: An Optimization Approach to Reconstructing Force-free Fields, ApJ, 540, 1150-1155, 2000.

Wiegelmann, T.: Optimization code with weighting function for the reconstruction of coronal magnetic fields, Solar Phys., 219, 87108, 2004.

Wiegelmann, T. and Inhester, B.: Magnetic Stereoscopy, Sol. Phys., 236, 25-40, doi:10.1007/s11207-006-0153-y, 2006.

Wiegelmann, T. and Neukirch, T.: Including stereoscopic information in the reconstruction of coronal magnetic fields, Solar Phys., 208, 233-251, 2002.

Wiegelmann, T. and Neukirch, T.: An optimization principle for the computation of MHD equilibria in the solar corona, A\&A, 457, 1053-1058, doi:10.1051/0004-6361:20065281, 2006.

Wiegelmann, T., Inhester, B., Lagg, A., and Solanki, S. K.: How To Use Magnetic Field Information For Coronal Loop Identification, Solar Phys., 228, 67-78, doi:10.1007/s11207-005-2511-6, 2005.

Wiegelmann, T., Inhester, B., and Sakurai, T.: Preprocessing of vector magnetograph data for a nonlinear force-free magnetic field reconstruction., Solar Phys., 233, 215-232, 2006.

Wiegelmann, T., Neukirch, T., Ruan, P., and Inhester, B.: Optimization approach for the computation of magnetohydrostatic coronal equilibria in spherical geometry, A\&A, 475, 701-706, doi:10.1051/0004-6361:20078244, 2007.

Wiegelmann, T., Thalmann, J. K., Schrijver, C. J., Derosa, M. L., and Metcalf, T. R.: Can We Improve the Preprocessing of Photospheric Vector Magnetograms by the Inclusion of Chromospheric Observations?, Solar Phys., 247, 249-267, doi:10.1007/ s11207-008-9130-y, 2008. 